\title{
miR-219-5p suppresses the proliferation and invasion of colorectal cancer cells by targeting calcyphosin
}

\author{
QUHUI WANG ${ }^{*}$, LIRONG ZHU* , YASU JIANG, JUNFEI XU, FEIRAN WANG and ZHIXIAN HE \\ Department of General Surgery, Affiliated Hospital of Nantong University, Nantong, Jiangsu 226001, P.R. China
}

Received December 5, 2015; Accepted October 27, 2016

DOI: $10.3892 / 01.2017 .5570$

\begin{abstract}
MicroRNAs (miRNAs) are small non-coding RNAs involved in an array of biological processes, and their dysregulation is associated with tumor development and progression. One such miRNA, miR-219-5p, is abnormally expressed in patients with colorectal cancer (CRC). In the present study, reverse transcription-quantitative polymerase chain reaction was performed to measure miR-219-5p expression in cells from both CRC tumors, and surrounding healthy tissue. MTT and invasion assays were used to determine the role of miR-219-5p in regulating $\mathrm{CRC}$ cell proliferation and invasion, respectively. A luciferase assay was then performed to assess the binding of miR-219-5p to the CAPS gene that encodes calcyphosin protein. The present study confirmed that miR-219-5p expression is significantly downregulated in CRC tissue. miR-219-5p knockdown promoted the growth of HCT-8 cells and increased the expression of calcyphosin protein (CAPS). On the other hand, overexpressing miR-219-5p inhibited HCT-8 cell growth and invasion, and downregulated CAPS expression. In addition, CAPS was identified as the functional downstream target of miR-219-5p by directly targeting its 3'-untranslated region. Therefore, miR-219-5p may function as a tumor suppressor by decreasing CAPS expression, and subsequently inhibit tumor proliferation and invasion. These results indicate that novel therapeutic strategies that increase miR-219-5p expression may be developed to treat CRC.
\end{abstract}

\section{Introduction}

Colorectal cancer (CRC) is one of the most common malignancies worldwide, and has multiple risk factors, associated with

Correspondence to: Dr Feiran Wang or Dr Zhixian He, Department of General Surgery, Affiliated Hospital of Nantong University, No. 20 Xisi Road, Nantong, Jiangsu 226001, P.R. China E-mail: wangfeirangs@sina.com

E-mail: hezhixiangs@sina.com

\section{*Contributed equally}

Key words: miR-219-5p, CAPS, colorectal cancer, tumor suppression lifestyle, genetics and the environment (1). Currently, the main therapeutic strategy used to treat CRC is surgical resection, however its effectiveness is limited in patients with locally invasive or metastatic disease. Therefore, understanding the mechanisms of CRC disease progression is essential in identifying potential biomarkers and improving CRC patient prognosis and treatment.

MicroRNAs (miRNAs) are small non-protein coding RNAs 20-24 nucleotides long, that negatively regulate gene expression by binding to the 3 ' untranslated region (3'UTR) of corresponding target messenger RNAs (mRNAs) (2). Abnormal miRNA expression is thought to contribute to tumorigenesis and carcinoma progression of various human cancers (3). Previous studies have demonstrated that analysis of the genes targeted by miRNA may identify altered miRNA regulatory networks involved in tumor pathogenesis; and miRNA itself may be used to identify cancer-specific signatures for CRC diagnosis (4). A number of studies have demonstrated that downregulating the expression of tumor suppressor miRNAs, such as miR-219-5p, may increase CRC development and progression (5). miR-219-5p inhibits papillary thyroid carcinoma cell growth and invasion by targeting estrogen receptor 1 (6), and targets glypican-3, inhibiting hepatocellular carcinoma cell proliferation (7). Furthermore, miR-219-5p regulates the receptor tyrosine kinase pathway in glioblastoma by targeting the epidermal growth factor receptor (8), and may regulate Sall4 in CRC cells (5).

Calcyphosine, a calcium binding protein of $24 \mathrm{kDa}$, was originally isolated from the canine thyroid cDNA library as a major phosphorylated substrate for protein kinase A following stimulation of thyroid cells by thyrotropin (8). As it is phosphorylated in a cAMP-dependent manner, it is considered to be implicated in the cross-signaling between these cascades to coordinate cellular proliferation and differentiation (9). Calcyphosine is also expressed in the brain, salivary glands and lungs of dogs (10). Moreover, calcyphosine functions as a candidate for cross-talk between cAMP-mediated and inositol trisphosphate $/ \mathrm{Ca}^{2+}$ mediated signal transduction pathways (11). Calcyphosine was thus identified as a tumor-specific protein that may serve as a tumor marker for a novel subgroup of ependymomas and as a potential drug target for therapy in pediatric brain tumors (12). The present study aims to identify a novel target gene of miR-219-5p and demonstrate that calcyphosin protein (CAPS) is targeted by miR-219-5p in human $\mathrm{CRC}$, to elucidate the mechanism by which miR-219-5p 
suppresses CRC progression. This would allow the clinical value of miR-219-5p in treating CRC patients to be evaluated.

\section{Materials and methods}

Tissue specimens and cell culture. Fresh CRC tissue specimens, and specimens from adjacent non-tumor tissues, were collected from 34 patients with CRC, who received surgical treatment at the Affiliated Hospital of Nantong University between May 2013 and May 2014. None of the patients had received prior neoadjuvant treatment. Samples were immediately frozen in liquid nitrogen, and stored at $-80^{\circ} \mathrm{C}$. All human tissue was collected using protocols approved by the Ethics Committee of Affliated Hospital of Nantong University.

Cell culture and transfection. Human ileocecal colorectal adenocarcinoma HCT-8 cells obtained from the Type Culture Collection of Chinese Academy of Sciences (Shanghai, China) were maintained in RPMI-1640 Medium (Gibco; Thermo Fisher Scientific, Inc., Waltham, MA, USA) supplemented with $10 \%$ fetal bovine serum (FBS, Invitrogen; Thermo Fisher Scientific, Inc.) in a humidified incubator with a mixture of $5 \% \mathrm{CO}_{2}$ at $37^{\circ} \mathrm{C}$. HCT-8 cells were then transfected with a scrambled miR-219-5p inhibitor (Thermo Fisher Scientific, Inc.), miR-219-5p mimics, or miRNA, which acted as a negative control (NC). For CAPS functional analysis, the coding sequence of CAPS was amplified and sub cloned into the pcDNA3.1 (+) vector (Invitrogen; Thermo Fisher Scientific, Inc.) according to the manufacturer instructions, in order to induce overexpression of endogenous CAPS. Following this, HCT-8 cells were transfected either with an empty vector or a CAPS-expressing plasmid, using lipofectamine 2000 (Invitrogen; Thermo Fisher Scientific, Inc.).

Reverse transcription-quantitative polymerase chain reaction $(R T-q P C R)$. Total RNA was extracted using a Trizol ${ }^{\circledR}$ Plus RNA purification kit (Invitrogen; Thermo Fisher Scientific, Inc), and cDNA was synthesized from total RNA treated with DNase I using an Omniscript reverse transcription kit (Qiagen Inc., Valencia, CA, USA) following the supplier's instructions. qPCR was performed using the HotStart-IT SYBR Green qPCR Master Mix (2X; USB Molecular Biology Reagents and Biochemicals; Thermo Fisher Scientific Inc.) to detect mRNA levels in CRC cells. According to the HotStart-IT protocol, $25 \mu \mathrm{l}$ reactions were run with $2 \mu \mathrm{l}$ cDNA. PCR experiments were performed in a LightCycler 480 system (Roche Diagnostics, Basel, Switzerland). The PCR procedure was as follows: Hot start at $95^{\circ} \mathrm{C}$ for $10 \mathrm{~min} ; 40$ cycles of amplification/quantification at $95^{\circ} \mathrm{C}$ for $10 \mathrm{sec}, 60^{\circ} \mathrm{C}$ for $30 \mathrm{sec}$, and $72^{\circ} \mathrm{C}$ for $30 \mathrm{sec}$ during which time fluorescence was measured. Melting curve analysis was performed using continuous fluorescence acquisition from $65-97^{\circ} \mathrm{C}$. According to the presence of a single melt peak, these cycling parameters generated single amplicons for both primer sets used. The sequences of the primers for CAPS were: Forward, 5'-AGGCACCTTCCACTAGCAACAG-3' and reverse, 5'-CCATGCTTGGTCTGGGCTCT-3'. The expression level of each target gene was normalized by the $\mathrm{Cq}$ value of U6 non-coding small nuclear RNA (U6), or glyceraldehyde 3-phosphate dehydrogenase (used as internal controls) using the $2^{-\Delta \Delta C t}$ relative quantification method (13).
For miRNA analysis, total RNA was extracted as described in the previous paragraph. Reverse transcription was performed using a miRNA 1st-Strand cDNA Synthesis kit (Stratagene; Agilent Technologies, Inc., Santa Clara, CA, USA). The cDNA were subjected to qPCR using the High-Specificity miRNA RT-qPCR Detection kit (Stratagene; Agilent Technologies, Inc.). The primers used were for hsa-miR-219-5p, and were as follows: Forward 5'-ACACTCCAGCTGGGTGATTGTCCA AACGC-3' and reverse, 5'-TGGTGTCGTGGAGTCG-3'; and U6, forward, 5'-CTCGCTTCGGCAGCACA-3' and reverse, 5'-AACGCTTCACGAATTTGCGT-3'. Finally, the relative miR-219-5P level was normalized to the endogenous reference gene U6 for each sample in triplicate, and was calculated by the $2^{-\Delta \Delta \mathrm{Cq}}$ relative quantification method (13).

Western blot analysis. Protein from tumor tissue was extracted by lysis buffer containing protease inhibitors. Wells were loaded with $10 \mu \mathrm{g} / \mathrm{well}$ of protein from each sample and then transferred to a polyvinylidene fluoride membrane. Blocking was subsequently carried out for $2 \mathrm{~h}$ using $5 \%$ non-fat milk in a mixture of Tris-buffered saline and Tween 20 (TBST). Following incubation with the primary monoclonal rabbit anti-human CAPS antibody (cat no. ab186741, 1:1,000, Abcam, Cambridge, MA, USA) overnight at $4^{\circ} \mathrm{C}$, the membranes were washed with TBST three times for $10 \mathrm{~min}$, and then incubated with horseradish peroxidase-conjugated goat anti-rabbit secondary antibody (cat no. ab97051, 1:5,000, Abcam) for $12 \mathrm{~h}$ at $4^{\circ} \mathrm{C}$. The ECL western blotting detection reagent (GE healthcare life sciences, Chalfont, UK) was used to detect signals prior to film development. $\beta$-actin was used as the loading control.

MTT assay. The MTT assay was used to determine the effect of miR-219-5p on cellular proliferation. A total of $1 \times 10^{3}$ cells containing $200 \mu \mathrm{l}$ RPMI-1640 supplemented with 10\% FBS were plated in each well of a 96-well plate. Following 1, 2, 3 days of incubation, $20 \mu 1$ MTT ( $5 \mathrm{mg} / \mathrm{ml}$; Sigma-Aldrich, St. Louis, MO, USA) was added, followed by $4 \mathrm{~h}$ incubation at $37^{\circ} \mathrm{C}$ in a $5 \% \mathrm{CO}_{2}$ incubator. The supernatant was subsequently removed and $150 \mu 1$ dimethyl sulfoxide was added. To dissolve MTT crystals, all plates were shaken for $10 \mathrm{~min}$ at room temperature. The absorbance values of each sample were read at $490 \mathrm{~nm}$ using a micro plate reader (Model 550, Bio-Rad Laboratories, Shanghai, China). Each experiment was repeated $\leq 3 \mathrm{x}$.

Colony formation assay. Following transfection of miR-219-5p mimics $(50 \mathrm{nmol} / \mathrm{l})$, miR-219-5p inhibitor $(50 \mathrm{nmol} / \mathrm{l})$ or scramble (NC; $50 \mathrm{nmol} / \mathrm{l}), \mathrm{HCT}-8$ cells were plated in a 24 -well plate for $24 \mathrm{~h}$. The cells were then collected and seeded $(1,000$ cells/well) in a fresh 6-well plate for 14 days. Surviving colonies ( $>50$ cells per colony) were counted following fixation with 1:1 methanol and acetone, and staining with $5 \%$ Gentian Violet (ICM Pharma Pte. Ltd., Singapore, Singapore). The experiment was carried out in triplicate.

Transwell assay. A transwell assay (BD Biosciences, Franklin Lakes, NJ, USA) was used to perform cell invasion according to the manufacturer's instructions. Mimics and inhibitors of miR-219-5p transfected cells were harvested $24 \mathrm{~h}$ following 
A

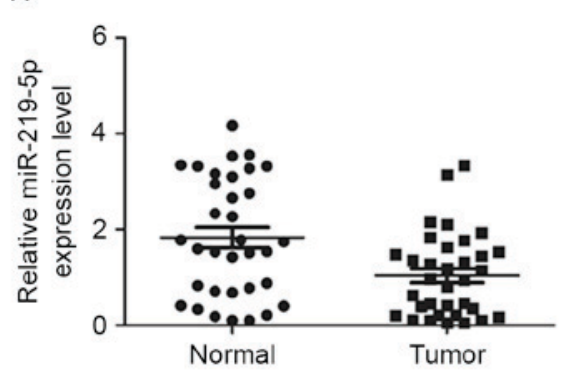

C
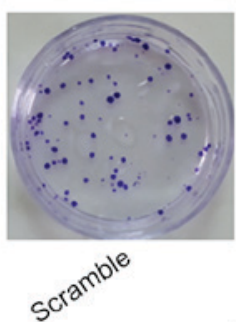
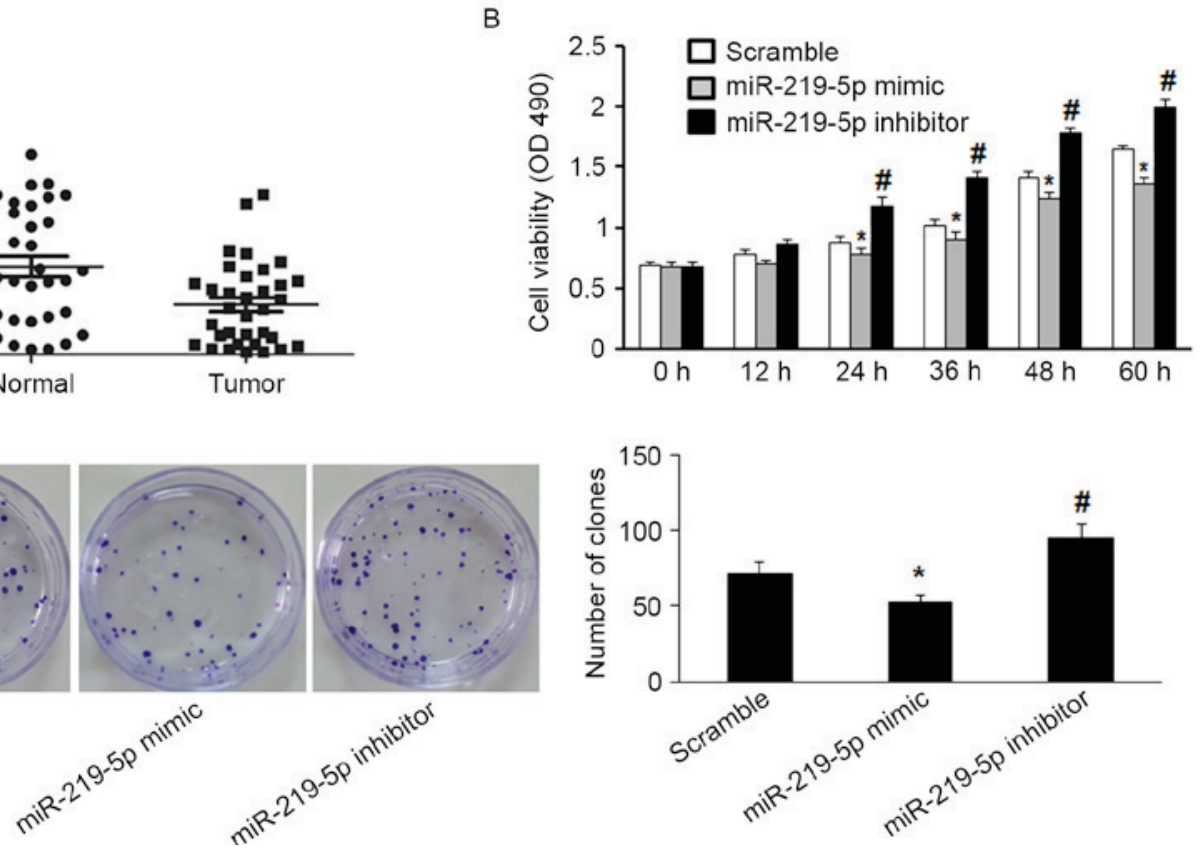

Figure 1. miR-219-5p inhibited the proliferation and growth of colorectal cancer (CRC) cells in vitro. (A) miR-219-5p expression was measured in the 34 samples of $\mathrm{CRC}$ tissues and matched adjacent non-tumor tissues by reverse transcription quantitative polymerase chain reaction (P=0.003). U6 snRNA was used as internal control. (B) The proliferation of human ileocecal colorectal adenocarcinoma HCT-8 cells transfected with miR-219-5p mimics, inhibitor, or scramble (control), was measured by MTT assay. (C) The number of colonies were evaluated by a colony formation assay of HCT-8 cells after treatment with miR-219-5p mimics, inhibitor, or scramble (control). ${ }^{*} \mathrm{P}<0.05$ miR-219-5p mimic vs. scramble; ${ }^{\#} \mathrm{P}<0.05$ miR-219-5p inhibitor vs. scramble.

transfection. $2.0 \times 10^{5}$ transfected cells or untreated cells in serum-free medium were then added to each upper insert pre-coated with Matrigel matrix, and $600 \mu 110 \%$ FBS medium was added to the matched lower chamber. Following incubation, non-invasive cells were removed from the upper surface of the Transwell membrane by a cotton swab. Invasive cells on the lower membrane surface were fixed with methanol, stained with $0.1 \%$ crystal violet, photographed, and counted.

Dual-luciferase reporter assay. HCT- 8 cells were seeded in 24-well plates $\left(1 \times 10^{5}\right.$ cells/well) and incubated for $24 \mathrm{~h}$ prior to transfection. For the reporter gene assay, the cells were co-transfected with either $0.5 \mu \mathrm{g}$ pGL3-CAPS-3'UTR wild-type or pGL3-CAPS-3'UTR mutant plasmid, $0.05 \mathrm{ng}$ pRL-TK control vector (Promega Corporation, Madison, WI, USA), and $50 \mathrm{nmol} / 1$ either miR-219-5p mimic or scramble, using Lipofectamine 2000 (Invitrogen; Thermo Fisher Scientific, Inc.). The firefly and renilla luciferase activities were measured consecutively through a dual luciferase assay (Promega Corporation) $24 \mathrm{~h}$ after transfection. Luciferase intensity was measured using the Dual Luciferase Reporter assay system (Promega Corporation,) according to the manufacturer instructions.

Statistical analysis. All statistical analyses were performed using SPSS software ver. 17.0 (SPSS, Inc., Chicago, IL, USA). Results were compared using either Student's $t$-test or $\chi^{2}$ test. One-way analysis of variance (ANOVA) test was used to analyze the significance of differences between groups of various differentiations. All data were presented as mean \pm standard deviation $(\mathrm{SD}) . \mathrm{P}<0.05$ was considered to indicate a statistically significant difference.

\section{Results}

Over-expression of miR-219-5p inhibits proliferation of CRC cells in vitro. RT-qPCR detected significantly decreased miR-219-5p expression in CRC tissue compared with the adjacent non-tumor tissue $(\mathrm{P}=0.003$, Fig. 1A). Moreover, the functional role of miR-219-5p in CRC cells was evaluated by measuring proliferation of HCT-8 cells. miR-219-5p expression was upregulated following transfection with miR-219-5p mimic, and downregulated by transfection with miR-219-5p inhibitor. The MTT assay was used to evaluate HCT- 8 cell proliferation. Transfection with miR-219-5p mimics suppressed cell proliferation while transfection with miR-219-5p inhibitor promoted cell proliferation (Fig. 1B). The inhibitory effect of miR-219-5p on the growth of CRC cells was confirmed by the colony formation assay; transfection with miR-219-5p mimic led to a significant reduction of colony numbers of HCT-8 cells compared with transfection with scramble (Fig. 1C, P<0.05). HCT-8 cells transfected with miR-219-5p inhibitor exhibited significantly increased cell proliferation compared with the control group (Fig. 1C, $\mathrm{P}<0.05$ ). These results demonstrate that miR-219-5p may function as a tumor suppressor in CRC carcinogenesis.

CAPS-3'UTR is a potential functional target of miR-219-5p. Previous studies have demonstrated that miR-219-5p suppresses the expression of several genes, such as Sall4, by targeting their 3'-UTR in CRC (5). To identify new potential target genes of miR-219-5p, the websites TargetScan (http://www.targetscan.org/) and miRanda (http://www.microrna.org/) were used. Bioinformatic analysis demonstrated that miR-219-5p directly targets the CAPS gene. 
A
Wild type of CAPS 3' UTR

has-miR-219-5P

Mutant of CAPS 3' UTR

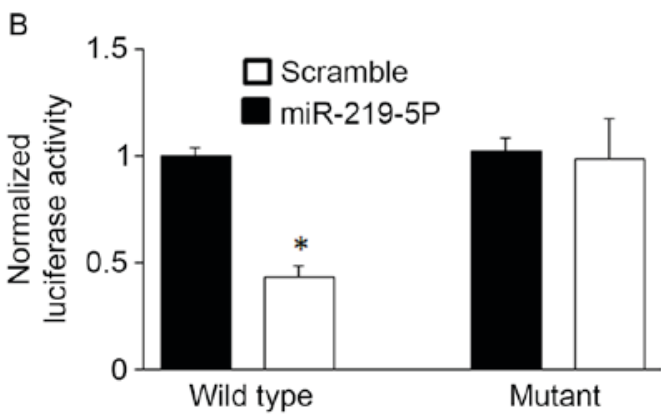

D

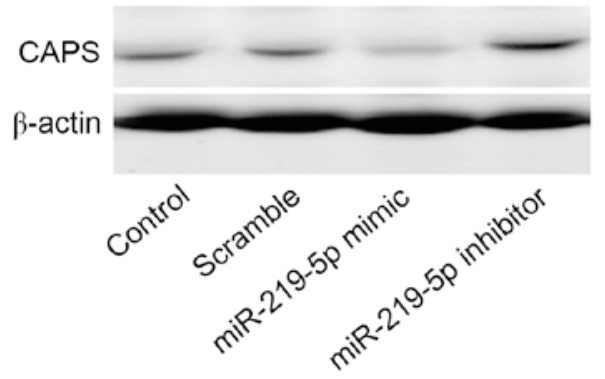

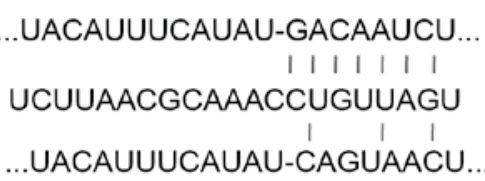
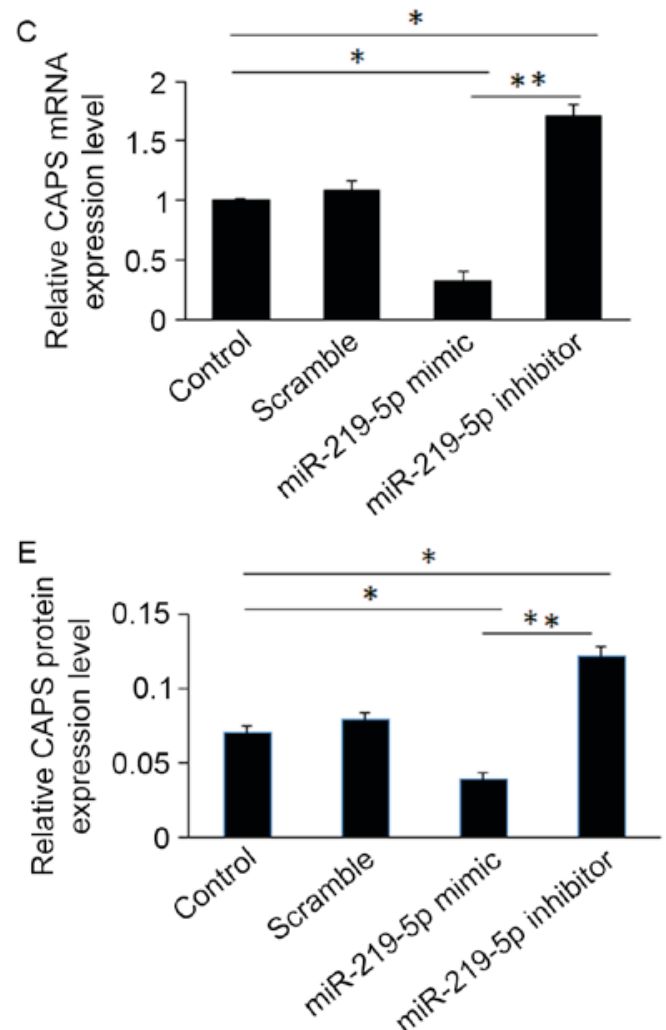

Figure 2. miR-219-5p downregulated calcyphosin protein (CAPS) expression through interaction with its 3' untranslated region (3'UTR). (A) Schematic representation indicated that wild-type (WT) CAPS 3'UTR was the putative miR-219-5p target site. (B) miR-219-5p significantly suppressed the luciferase activity in WT but not mutant type (MUT) 3'UTR of CAPS. Firefly luciferase values were normalized to Renilla luciferase activity and plotted as relative luciferase activity. (C-E) Reverse transcription quantitative polymerase chain reaction and western blot analysis were performed to examine the effects of miR-219-5p on CAPS expression in human ileocecal colorectal adenocarcinoma HCT-8 cells transfected with miR-219-5p mimics, miR-219-5p inhibitor, no transfection (control) or negative control (scramble). ${ }^{*} \mathrm{P}<0.05,{ }^{* *} \mathrm{P}<0.001$.

To validate this, the wild-type and mutant sequences of the CAPS 3'UTR were cloned into luciferase reporter vectors (sequences shown in Fig. 2A). The luciferase assay revealed that miR-219-5p significantly suppressed luciferase activity of CAPS containing a wild-type 3'UTR, however it did not suppress the activity of CAPS containing a mutant 3'UTR $(\mathrm{P}<0.05$, Fig. 2B). This confirms that miR-219-5p targets the 3'UTR of CAPS.

miR-219-5p mimics significantly decreased CAPS protein and mRNA levels compared to controls, whereas miR-219-5p inhibitors increased levels of CAPS mRNA and protein in HCT-8 cells (Figs. 2C and E). This was confirmed by western blot analysis (Fig. 2D).

Furthermore, the mRNA level of CAPS in the 34 pairs of tumor and adjacent non-tumor tissue were analyzed by RT-qPCR. CAPS mRNA levels were significantly higher in $\mathrm{CRC}$ tissue than in healthy tissue $(\mathrm{P}<0.001$, Fig. 3A). Furthermore, a significant negative correlation was observed between miR-219-5p and CAPS expression in CRC samples $\left(r^{2}=-0.47\right.$, $\mathrm{P}<0.001$, Fig. 3B). Taken all together, the results of the current study suggest that miR-219-5p decreases the expression of CAPS in CRC by directly targeting CAPS 3'UTR.
Restoration of miR-219-5p inhibits CAPS-mediated CRC cell invasion. Western blot analysis indicated that HCT-8 cells transfected with the pcDNA3.1 (+)-CAPS may enhance CAPS protein expression compared with cells transfected with an empty vector control (Fig. 4A). Furthermore, the Transwell assay demonstrated that HCT-8 cells transfected with miR-219-5p mimic and pcDNA3.1 (+)-CAPS overexpression of miR-219-5p $(312 \pm 12.46)$ exhibited significantly decreased migratory capacity compared with CAPS-induced CRC cells transfected with CAPS and a scramble (403 \pm 18.53 ; Fig. 4B, $\mathrm{P}<0.05)$.

\section{Discussion}

Recently, the role of miRNAs in tumorigenesis and their regulatory function in a number of biological processes associated with cancer has been investigated. In human cancer, miRNAs are usually located in genomic breakpoint regions and function as tumor suppressor genes or oncogenes during tumor development and progression (14). miR-219-5p may act as a tumor manipulator in various types of cancers by targeting downstream oncogenic molecules, thus attenuating 
A

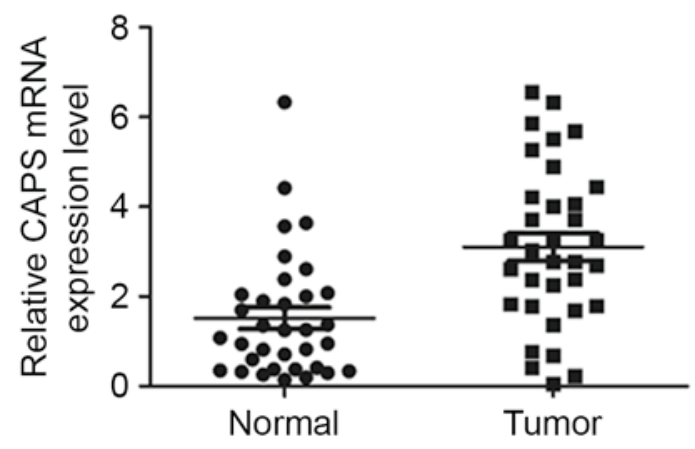

B

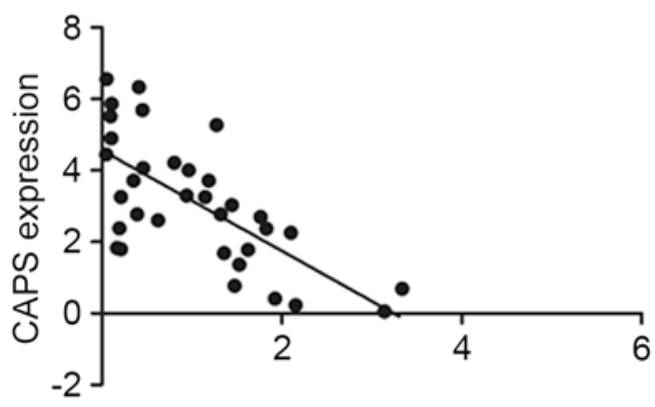

miR-219-5p expression

Figure 3. Calcyphosin protein (CAPS) expression was inversely correlated with miR-219-5p levels in colorectal cancer (CRC) cells. (A) CAPS expression was measured in $34 \mathrm{CRC}$ tissues and matched adjacent non-tumor tissues by reverse transcription quantitative polymerase chain reaction ( $\mathrm{P}<0.001)$. Glyceraldehyde 3-phosphate dehydrogenase was used as an internal control. (B) The association between CAPS and miR-219-5p expression was explored by Spearman's correlation analysis in CRC cells $\left(\mathrm{r}^{2}=-0.47, \mathrm{P}<0.001\right)$.

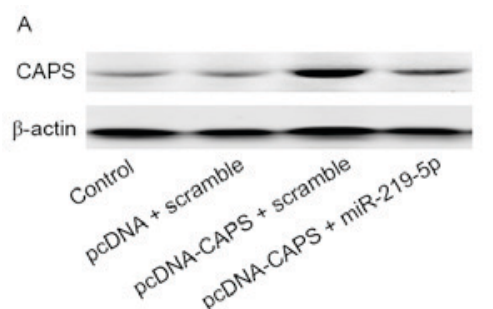

B

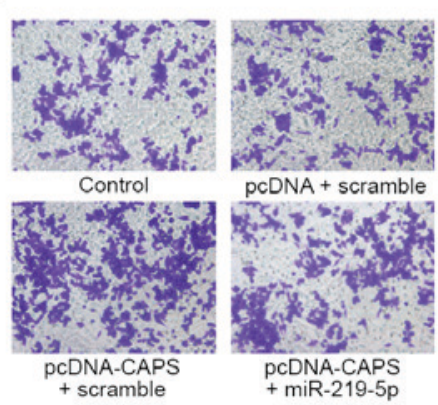

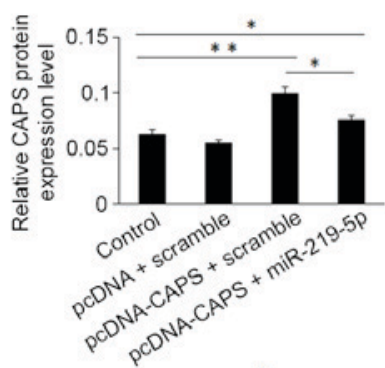

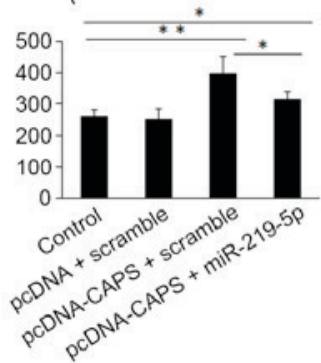

Figure 4. Restoration of miR-219-5p inhibits the Calcyphosin protein (CAPS)-mediated colorectal cancer cell invasion. (A) Western blotting was performed to examine the levels of CAPS protein in human ileocecal colorectal adenocarcinoma HCT-8 cells co-transfected with either miR-219-5p mimic or scramble and pcDNA-CAPS or pcDNA empty vector using an MTT assay. (B) Cell invasion in HCT-8 cells co-transfected with either miR-219-5p mimic or scramble and pcDNA-CAPS or pcDNA empty vector using invasion assay was analyzed. ${ }^{*} \mathrm{P}<0.05,{ }^{* *} \mathrm{P}<0.001$.

the malignant features of hepatocellular carcinoma (7), papillary thyroid carcinoma (6), glioblastoma (15) and colorectal cancer (5).

In the present study, analysis by RT-qPCR demonstrated that miR-219-5p is downregulated in CRC tissue compared with adjacent non-tumor tissues. Evidence gathered from numerous studies highlights the significance of miRNAs as being vital in the regulation of numerous physiological processes such as cell proliferation, differentiation and apoptosis (16-18). Both the MTT and colony formation assays demonstrated that miR-219-5p significantly suppressed the growth and proliferation of CRC cells in vitro. By contrast, downregulating miR-219-5p expression promoted CRC cell proliferation.

Numerous studies have indicated that miRNAs are deregulated in $\mathrm{CRC}$, and the importance of this deregulation is highlighted by the importance of the target genes they regulate (19-21). The current study sought to identify other potential targets of miR-219-5p to further understand how miR-219-5p suppresses tumors in CRC. Target prediction algorithms identified the binding sites for miR-219-5p in the 3'-UTR of CAPS.

CAPS belongs to the calmodulin superfamily of calcium-binding proteins and is regulated by cyclic AMP through protein phosphorylation (22). It is phosphorylated in a cAMP-dependent manner and thus may be implicated in the cross-signaling between these cascades to coordinate cell proliferation and differentiation (9), suggesting its involvment in carcinogenesis. It has been proven that CAPS is expressed in many tumors; overexpression of CAPS occurs in ependymoma (23), lung cancer (24), ovarian cancer (25) and endometrial cancer (26).

In conclusion, the present study identified CAPS as a direct target of miR-219-5p in CRC cells. CAPS was upregulated 
in CRC tissues, and miR-219-5p expression in CRC tissues was inversely correlated with CAPS expression: Increasing miR-219-5p expression downregulated the expression of CAPS protein, while knockdown of miR-219-5p increased CAPS protein levels. Furthermore, CAPS-induced cell invasion was reversed by miR-219-5p expression. Therefore, miR-219-5p may function as a tumor suppressor and inhibit tumor proliferation and invasion by regulating CAPS expression.

\section{Acknowledgements}

The present study was supported by the Affiliated Hospital of Nantong University (grant no. TDFzh201413).

\section{References}

1. Siegel R, Naishadham D and Jemal A: Cancer statistics, 2013. CA Cancer J Clin 63: 11-30, 2013.

2. Bartel DP: MicroRNAs: Genomics, biogenesis, mechanism, and function. Cell 116: 281-297, 2004.

3. Iorio MV and Croce CM: MicroRNAs in cancer: Small molecules with a huge impact. J Clin Oncol 27: 5848-5856, 2009.

4. Sun Y, Wang L, Guo SC, Wu XB and Xu XH: High-throughput sequencing to identify miRNA biomarkers in colorectal cancer patients. Oncol Lett 8: 711-713, 2014.

5. Cheng J, Deng R, Zhang P, Wu C, Wu K, Shi L, Liu X, Bai J, Deng M, Shuai X, et al: miR-219-5p plays a tumor suppressive role in colon cancer by targeting oncogene Sall4. Oncol Rep 34: 1923-1932, 2015.

6. Huang C, Cai Z, Huang M, Mao C, Zhang Q, Lin Y, Zhang X, Tang B, Chen Y, Wang X, et al: miR-219-5p modulates cell growth of papillary thyroid carcinoma by targeting estrogen receptor $\alpha$. J Clin Endocrinol Metab 100: E204-E213, 2015.

7. Huang N, Lin J, Ruan J, Su N, Qing R, Liu F, He B, Lv C, Zheng D and Luo R: MiR-219-5p inhibits hepatocellular carcinoma cell proliferation by targeting glypican-3. FEBS Lett 586: 884-891, 2012.

8. Lecocq R, Lamy F and Dumont JE: Pattern of protein phosphorylation in intact stimulated cells: Thyrotropin and dog thyroid. Eur J Biochem 102: 147-152, 1980.

9. Clément S, Dumont JE and Schurmans S: Loss of calcyphosine gene expression in mouse and other rodents. Biochem Biophys Res Commun 232: 407-413, 1997.

10. Lefort A, Lecocq R, Libert F, Lamy F, Swillens S, Vassart G and Dumont JE: Cloning and sequencing of a calcium-binding protein regulated by cyclic AMP in the thyroid. Embo J 8: 111-116, 1989.
11. Nemoto Y, Ikeda J, Katoh K, Koshimoto H, Yoshihara Y and Mori K: R2D5 antigen: A calcium-binding phosphoprotein predominantly expressed in olfactory receptor neurons. J Cell Biol 123: 963-976, 1993

12. Dong H, Li X, Lou Z, Xu X, Su D, Zhou X, Zhou W, Bartlam M and Rao Z: Crystal-structure and biochemical characterization of recombinant human calcyphosine delineates a novel EF-hand-containing protein family. J Mol Biol 383: 455-464, 2008.

13. Livak KJ and Schmittgen TD: Analysis of relative gene expression data using real-time quantitative PCR and the 2(-Delta Delta C(T)) Method. Methods 25: 402-408, 2001.

14. Garzon R, Calin GA and Croce CM: MicroRNAs in Cancer. Annu Rev Med 60: 167-179, 2009.

15. Jiang Y, Yin L, Jing H and Zhang H: MicroRNA-219-5p exerts tumor suppressor function by targeting ROBO1 in glioblastoma. Tumour Biol 36: 8943-8951, 2015.

16. Sen R, Ghosal S, Das S, Balti S and Chakrabarti J: Competing endogenous RNA: The key to posttranscriptional regulation. ScientificWorldJournal 2014: 896206, 2014.

17. Nicoloso MS, Spizzo R, Shimizu M, Rossi S and Calin GA: MicroRNAs-the micro steering wheel of tumour metastases. Nat Rev Cancer 9: 293-302, 2009.

18. Esquela-kerscher A and Slack FJ: Oncomirs - microRNAs with a role in cancer. Nat Rev Cancer 6: 259-269, 2006.

19. Ma Y, Zhang P, Wang F, Zhang H, Yang J, Peng J, Liu W and Qin H: miR-150 as a potential biomarker associated with prognosis and therapeutic outcome in colorectal cancer. Gut 61: 1447-1453, 2012.

20. Schee K, Fodstad $\varnothing$ and Flatmark K: MicroRNAs as biomarkers in colorectal cancer. Am J Pathol 177: 1592-1599, 2010.

21. He X, Dong Y, Wu CW, Zhao Z, Ng SS, Chan FK, Sung JJ and Yu J: MicroRNA-218 inhibits cell cycle progression and promotes apoptosis in colon cancer by downregulating BMI1 polycomb ring finger oncogene. Mol Med 18: 1491-1498, 2013.

22. Kretsinger RH: Crystallographic studies of calmodulin and homologs. Ann N Y Acad Sci 356: 14-19, 1980.

23. de Bont JM, de BoerML, Kros JM, Passier MM, Reddingius RE, Smitt PA, Luider TM and Pieters R: Identification of novel biomarkers in pediatric primitive neuroectodermal tumors and ependymomas by proteome-wide analysis. J Neuropathol Exp Neurol 66: 505-516, 2007.

24. Pastor MD, Nogal A, Molina-Pinelo S, Meléndez R, Salinas A, González De la Peña M, Martín-Juan J, Corral J, García-Carbonero R, Carnero A and Paz-Ares L: Identification of proteomic signatures associated with lung cancer and COPD. J Proteomics 89: 227-237, 2013.

25. Partheen K, Levan K, Osterberg L and Horvath G: Expression analysis of stage III serous ovarian adenocarcinoma distinguishes a sub-group of survivors. Eur J Cancer 42: 2846-2854, 2006.

26. Li Z, Min W, Huang $C$, Bai S, Tang $M$ and Zhao $X$ : Proteomics-based approach identified differentially expressed proteins with potential roles in endometrial carcinoma. Int J Gynecol Cancer 20: 9-15, 2010. 